\title{
Clinical manifestations and treatment of mucopolysaccharidosis type I patients in Latin America as compared with the rest of the world
}

\author{
María Verónica Muñoz-Rojas • Luisa Bay • \\ Luz Sanchez • Marcel van Kuijck • Sandra Ospina • \\ Juan Francisco Cabello • Ana Maria Martins
}

Received: 19 January 2011 /Revised: 31 March 2011 / Accepted: 5 April 2011 /Published online: 4 May 2011

(C) The Author(s) 2011. This article is published with open access at Springerlink.com

\begin{abstract}
Background Mucopolysaccharidosis I (MPS I) comprises a spectrum of clinical manifestations and is divided into three phenotypes reflecting clinical severity: Hurler, HurlerScheie, and Scheie syndromes. There may be important variations in clinical manifestations of this genetic disease in patients residing in different regions of the world.

Methods Using data from the MPS I Registry (as of September 2009), we evaluated patients from Latin America $(n=118)$ compared with patients from the rest of the world [ROW $(n=727)]$.

Results Phenotype distribution differed among patients in Latin America compared to ROW (Hurler 31 vs. 62\%, HurlerScheie 36 vs. $21 \%$, Scheie 10 vs. $11 \%$, and unknown 22 vs. $6 \%$ ). The frequency of certain symptoms, such as cardiac
\end{abstract}

Communicated by: Ed Wraith

M. V. Muñoz-Rojas $(\bowtie)$

Genzyme do Brazil,

Av. Francisco Matarazzo, 1400, Edifício Milano - $10^{\circ}$ andar, 05001-903 São Paulo, SP, Brazil

e-mail: veronica.munoz@genzyme.com

\section{Bay}

Unidad de Errores Congénitos del Metabolismo,

Hospital Nacional de Pediatría J.P. Garrahan,

Buenos Aires, Argentina

L. Sanchez

Pediatra y Neonatóloga, Hospital de Especialidades UMAE 25,

Monterrey, México

M. van Kuijck

Registry \& Compassionate Use Programs, Genzyme, Latin America Group,

Rio de Janeiro, Brazil valve abnormalities, sleep impairment, and joint contractures, also differed between Latin America and ROW for some phenotypes. Median age at MPS I diagnosis was earlier in the ROW than Latin America for all phenotypes, and age at first treatment for Hurler and Hurler-Scheie patients was also earlier in the ROW. Hurler patients in Latin America showed a gap of 3.1 years between median ages of diagnosis and first treatment compared to only 0.5 years in the ROW. Treatment allocation in Latin America compared to ROW was as follows: enzyme replacement therapy (ERT) only, 80 vs. 45\%; hematopoietic stem cell transplantation (HSCT) only, 0.9 vs. $27 \%$; both ERT and HSCT, 0 vs. $16 \%$; and neither treatment, 19 vs. $13 \%$.

Conclusion These data highlight important differences in MPS I patients between Latin America and ROW in terms

\section{S. Ospina}

Fundación Universitaria de Ciencias de la Salud,

Universidad del Rosario,

Bogotá, Colombia

J. F. Cabello

Neurólogo Infantil. Programa de Formación Neuropediatria,

Facultad de Medicina, Univerisad de Valparaiso,

Valparaiso, Chile

J. F. Cabello

Laboratorio de Genética y Enfermedades Metabólicas del Instituto de Nutrición y Tecnología de los Alimentos (INTA),

Universidad de Chile,

Santiago, Chile

A. M. Martins

Centro de Referência em Erros Inatos do Metabolismo,

Universidade Federal de São Paulo,

São Paulo, Brazil 
of phenotypic distribution, clinical manifestations, and treatment practices.

\section{Introduction}

Mucopolysaccharidosis type I (MPS I; OMIM \#252800) is a rare condition, inherited in an autosomal recessive manner. It is a lysosomal storage disorder caused by a deficiency of alpha-L-iduronidase (IDUA) enzymatic activity, which in turn results in the accumulation of the glycosaminoglycans (GAGs) heparan sulfate and dermatan sulfate in organs and systems, ultimately leading to multisystemic cellular and organ dysfunction (Neufeld and Muenzer 2001; Valstar et al. 2008). MPS I encompasses a spectrum of clinical manifestations and is usually divided into three phenotypes by clinical severity: Hurler, HurlerScheie, and Scheie syndrome. Patients with Hurler syndrome develop symptoms in early childhood and have marked cognitive delay (Neufeld and Muenzer 2001; Staba et al. 2004; Wraith et al. 2005). Without treatment, life expectancy is usually limited to the first decade (Neufeld and Muenzer 2001; Pastores et al. 2007; Muenzer et al. 2009). At the other end of the spectrum, patients with Scheie syndrome present with symptoms much later in childhood and adolescence and show slower disease progression. They have normal intelligence and survive into adulthood, although they usually experience important disease-related morbidity (Neufeld and Muenzer 2001; Vijay and Wraith 2005; Thomas et al. 2006, 2010; Pastores et al. 2007). Patients whose features are intermediate between Hurler and Scheie syndromes are usually classified as having Hurler-Scheie syndrome, which is generally characterized by mild or no cognitive impairment (Neufeld and Muenzer 2001; Pastores et al. 2007) and relatively severe somatic symptoms limiting life expectancy to the second or third decade in the absence of treatment (Pastores et al. 2007; Muenzer et al. 2009). However, this traditional subtype classification is not based on precisely defined criteria and is not interpreted in a consistent fashion by all practitioners (Pastores et al. 2007).

Currently two non-mutually exclusive therapeutic options are available for MPS I: hematopoietic stem cell transplantation (HSCT) and enzyme replacement therapy (ERT) with laronidase (recombinant human $\alpha$-Liduronidase; Aldurazyme ${ }^{\mathcal{O}}$, Genzyme) (Cox-Brinkman et al. 2006; Boelens et al. 2007; Pastores et al. 2007; Muenzer et al. 2009). Cellular therapy through either derived bone marrow or umbilical cord donor stem cells can induce a remarkable change in the clinical course; reported transplant outcomes include stabilization of neurocognitive function if performed at a relatively early age - generally before the age of 2 years - as well as prevention of fatal cardiopulmonary complications and improvement in overall survival (Cox-Brinkman et al. 2006; Boelens et al. 2007; Aldenhoven et al. 2008). However, HSCT has little effect on skeletal problems (Staba et al. 2004; Khanna et al. 2007; Aldenhoven et al. 2008) and carries a significant procedural risk (Cox-Brinkman et al. 2006; Aldenhoven et al. 2008). The more recently developed ERT with laronidase (Wraith et al. 2005; Wraith 2005) has been shown to improve respiratory and cardiac symptoms and some skeletal and joint manifestations, to reduce hepatosplenomegaly, and to improve overall quality of life (Wraith et al. 2004; Wraith 2005; Sifuentes et al. 2007; Clarke et al. 2009). However, ERT with laronidase at the labelled dose, $0.58 \mathrm{mg} / \mathrm{kg}$, is not expected to prevent cognitive decline as the blood-brain barrier prevents the intravenously delivered enzyme from reaching the central nervous system in significant amounts (Kakkis et al. 2001; Wraith et al. 2005; Pastores et al. 2007). Early diagnosis and determination of phenotype are therefore of major importance to determine the best treatment option for each MPS I patient.

There are many variations in the clinical manifestations of MPS I among individual patients, and there may also be important differences in the clinical manifestations of patients residing in different regions of the world. In the present report, we undertook a study through the MPS I Registry, the largest registry that tracks clinical data for patients with MPS I, to characterize the phenotypes of patients from the Latin America region and to compare them with those of patients from the rest of the world (ROW). The MPS I Registry (www.mpsiregistry.com) is an international, observational, voluntary program initiated in 2003 to track treatment patterns and clinical status of patients with MPS I over time in order to increase understanding and awareness of this disease.

\section{Methods}

We characterized the phenotypes of patients enrolled in the MPS I Registry from the Latin America region (all countries from South America plus Mexico) and compared them with those of patients in the Registry from the rest of the world (ROW). All patients with MPS I are eligible to participate in the MPS I Registry regardless of whether the patient is receiving any treatment and independent of the type of treatment the patient is receiving. There are no exclusion criteria, all that is needed is a diagnosis confirmation and a signed patient consent. While the focus of the Registry is on prospective data capture, patients may be enrolled posthumously in order to enhance the understanding of the natural history of MPS I.

Herein reported analyses were conducted using data available as of September 2009. The data analyzed were the 
number of patients enrolled in each region, phenotype distribution, the most frequent symptoms, age at onset of symptoms, age at diagnosis, age at initiation of treatment, and type of treatment. Numerical comparisons were made, but formal statistical evaluations were not performed and no $P$-values were generated for these comparisons.

The MPS I Registry Program has the approval of ethics committees. A specific ethics approval for this paper was not required; supporting documents are available upon request.

\section{Results}

As of September 2009, 845 patients were enrolled in the MPS I Registry worldwide. A total of 118 patients were enrolled from five countries in Latin America (60\% Brazil, 14\% Argentina, 17\% Mexico, 3\% Chile, and 5\% Colombia) as shown in Fig. 1. The ROW patient group totaled 727 patients from 27 countries: $56 \%$ from Europe, $41 \%$ from North America, and 3\% from Asia Pacific. Patients from Brazil made up the majority of Latin American patients. The phenotype distribution for Latin American patients and patients from the ROW is presented in Fig. 2a. The distribution of phenotypes for Registry patients in these two regions appeared to be different, with $31 \%$ of Latin American patients reported to have Hurler syndrome, while in ROW patients Hurler was the most common phenotype accounting for $62 \%$ of patients. Overall there was a higher proportion of patients in Latin America for whom the phenotype was undetermined or unreported (22 versus $6 \%$ in the ROW). Figure $2 \mathrm{~b}$ displays the phenotype distribution of patients enrolled in Latin America stratified by country. Hurler was the most common phenotype reported in patients in Argentina, Colombia, and Mexico, while Hurler-Scheie syndrome accounted for the majority of patients with MPS I disease in Brazil and Chile. However,

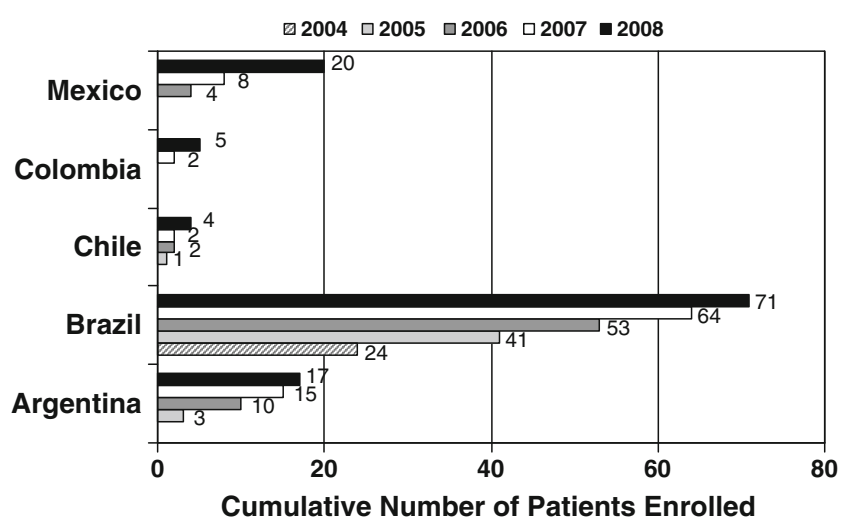

Fig. 1 Number of patients enrolled in MPS I Registry in Latin America by country and year it should be kept in mind that there is a fairly large percentage of patients with missing or undetermined phenotype, and the sample sizes for some countries such as Chile and Colombia are quite small.

Phenotype classification is expected to be related to the age at onset of MPS I symptoms, although there are many other contributing factors. The median ages at onset of MPS I-related symptoms are summarized in Fig. 3. This figure shows similar ages at symptom onset for the Hurler patients, Scheie patients, and patients with an undetermined or missing phenotype in Latin America compared to ROW. However, the Hurler-Scheie patients in Latin America seemed to have a younger age at symptom onset than the Hurler-Scheie patients in the ROW (1 year versus 2.2 years). The median ages at MPS I diagnosis and first treatment (ERT or HSCT) by phenotype are also presented in Fig. 3. Hurler patients in Latin America showed a delay of 3.1 years between the median age of diagnosis and the median age of first treatment, while Hurler patients in the ROW were generally treated earlier, with a corresponding delay of only 0.5 years. Conversely, Scheie patients in Latin America showed a delay of 8.5 years from diagnosis to first treatment, while in the ROW the delay was 5.7 years. Registry data regarding laronidase treatment regimens were also analyzed. No major differences in dose, dose frequency, or infusion time between Latin American and ROW patients were apparent. Most patients in both regions were reported to receive laronidase at the dose recommended on the drug label $(0.58 \mathrm{mg} / \mathrm{kg}$ intravenously once a week).

The symptom profile in MPS I is expected to differ across phenotypes, but not necessarily by region. Figure 4 displays the frequencies of key MPS I signs and symptoms reported in patients at the time of enrollment in the Registry, according to phenotype and region. Only those signs reported in at least $40 \%$ of Latin American patients, by phenotype, are presented. Several apparent regional differences in symptom frequency were seen. In Hurler patients, joint contractures were reported in $97 \%$ of Latin America patients but in only $52 \%$ of patients in the ROW. Other clinical findings reported more frequently in Latin American Hurler patients compared to ROW included cognitive impairment (89 versus 62\%), sleep disturbance (89 versus 64\%), enlarged tongue ( 89 versus $49 \%$ ), dysostosis multiplex (81 versus 60\%), and enlarged tonsils (68 versus 39\%). Conversely, cardiac valve abnormalities were reported in $73 \%$ of ROW Hurler patients but in only $35 \%$ of Latin American Hurler patients. In Hurler-Scheie patients, differences in symptom frequency were slightly less apparent. Joint contracture was reported in $90 \%$ of Latin American patients but in only $78 \%$ of patients in the ROW, and pneumonia was reported in $48 \%$ of Latin American patients compared to only $21 \%$ of ROW patients. Conversely, cardiac valve abnormalities were reported in 
Fig. 2 a MPS I phenotype distribution for Latin American patients and patients from the rest of the world (ROW). b Phenotype distribution of Latin American enrolled MPS I patients by country
A
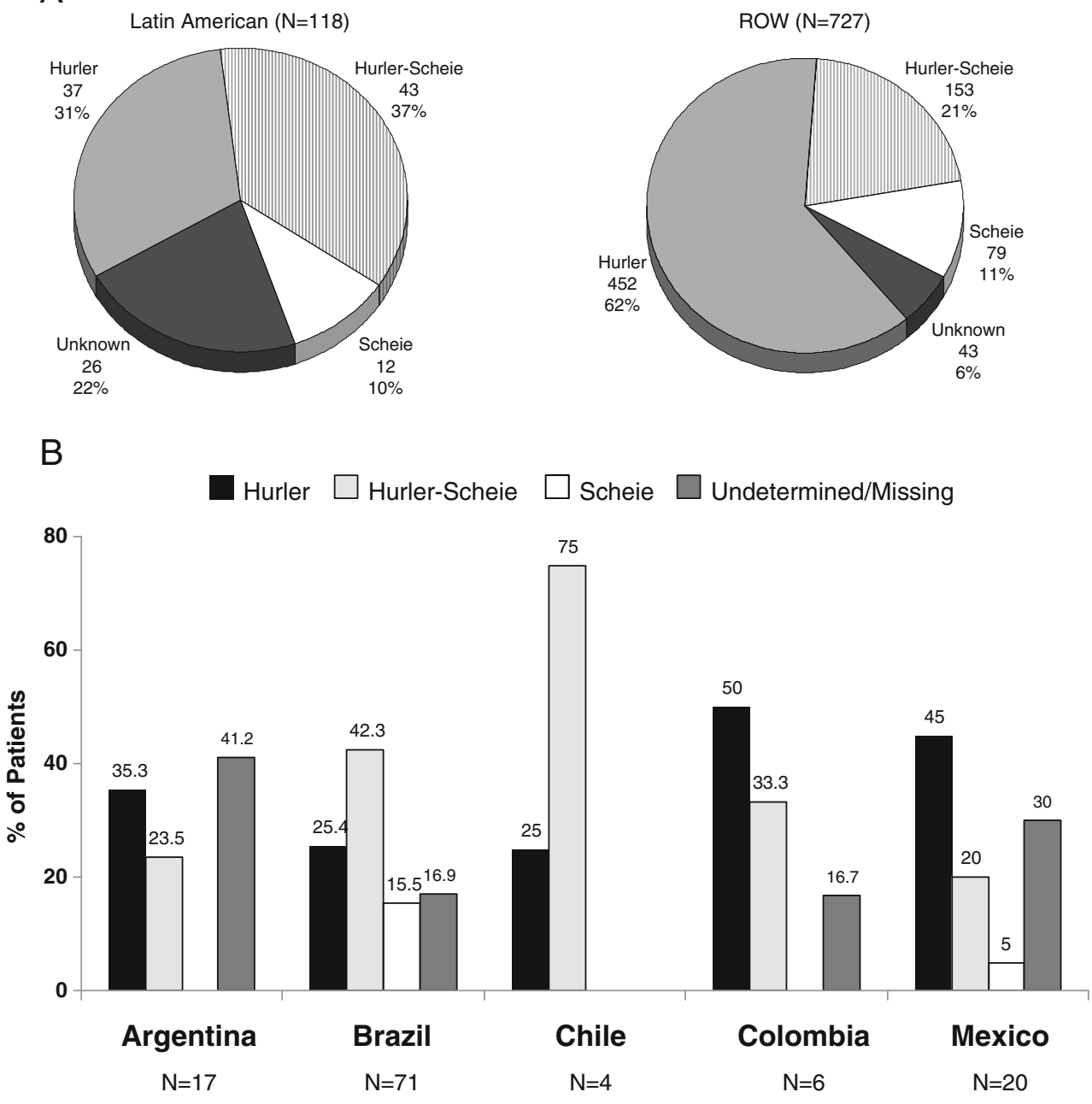

$87 \%$ of ROW but in only $54 \%$ of Latin American HurlerScheie patients. A similar frequency of cognitive impairment was reported in 33\% of Latin American and 35\% of ROW Hurler-Scheie patients. In Scheie patients, the symptoms with the largest regional difference in frequency were sleep disturbance (75\% Latin America, 42\% ROW), cardiac valve abnormalities (67\% Latin America, 95\% ROW), and hepatomegaly (83\% Latin America, 55\% ROW). Among patients with an undetermined or missing phenotype, differences in frequency by region of $15 \%$ or more were noted for most symptoms with the exception of enlarged tongue, corneal clouding, sleep disturbances/ snoring, scoliosis, and kyphosis/gibbus, each of which occurred in a similar percentage of patients in Latin America and the ROW. Such variation is likely due to a wide array of phenotypes in this group. Of note, the frequency of cognitive impairment was lower in Latin American compared to ROW patients with an undetermined or missing phenotype (31 versus 53\%).

Of the 118 enrolled patients in Latin America, 80\% were reported to have received ERT only, $0.9 \%$ to have received
HSCT only, 0\% both ERT and HSCT, and 19\% were reported to have received neither HSCT nor ERT. Of 727 Registry patients in the ROW, $45 \%$ received ERT only, 27\% received HSCT only, $16 \%$ received both ERT and HSCT, and $13 \%$ received neither treatment. Treatment status by phenotype is presented in Fig. 5. This analysis revealed that $63 \%$ of Hurler patients in the ROW received HSCT with or without ERT, while in Latin America the primary treatment for Hurler patients was ERT alone (83\% of Latin American Hurler patients).

\section{Discussion}

The MPS I Registry has emerged as the largest database for monitoring the clinical progression of MPS I disease worldwide. The design and methodology of the program have enabled the acquisition of a population sample that is diverse in its composition and representative of the heterogeneous nature of MPS I (Pastores et al. 2007; Thomas et al. 2010). The data presented here give the Latin 


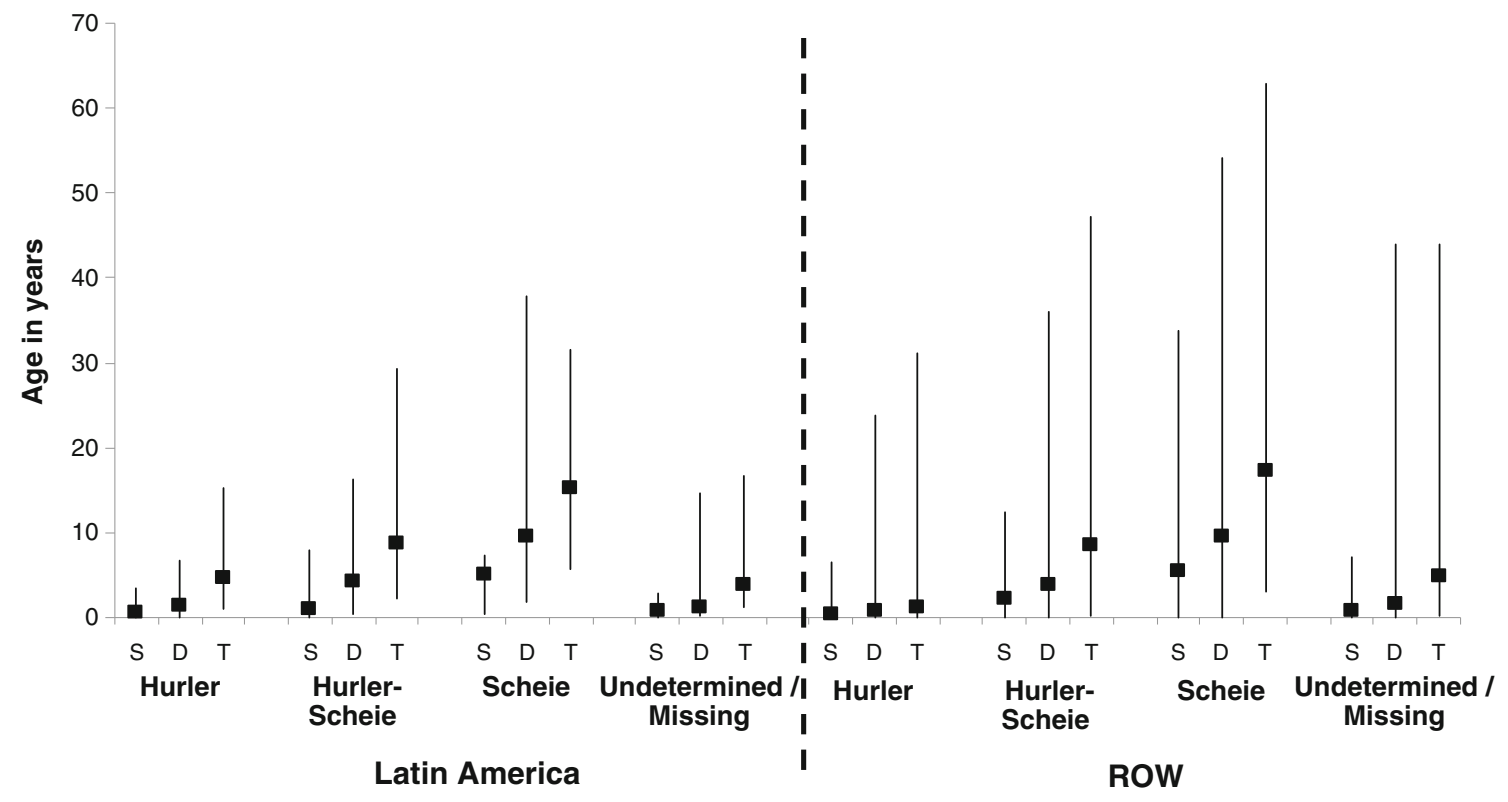

$\mathrm{S}=$ Age at symptom onset; $\mathrm{D}=$ Age at MPS I diagnosis; $\mathrm{T}=$ Age at $1^{\text {st }}$ treatment

Black boxes indicate median ages while black lines represent the range (min,max)

Fig. 3 Median age of symptom onset of MPS I-related symptoms, diagnosis, and treatment initiation by region and phenotype

American population numbers for several parameters regarding disease onset, symptomatology, and treatment decision options.

The enrollment data suggest a different MPS I phenotype distribution in Latin America compared to ROW, with Latin America reporting a smaller proportion of Hurler patients (31 versus $62 \%$ ) and a higher proportion of Hurler-Scheie patients (37 versus $21 \%$ ) and patients with an unknown or undetermined diagnosis (22 versus 6\%). There also appeared to be phenotype distribution differences among the individual Latin American countries. However, the sample size in Latin America was relatively small $(n=118)$, and therefore caution should be used when interpreting these data. In addition, $60 \%$ of patients enrolled from Latin America were from Brazil, and the majority of observed trends are likely to be driven by this subgroup. The remaining $40 \%$ of patients were widely distributed among the other Latin American countries, making it impossible to assess more definitive differences among patients from the different countries.

Some of the differences observed between Latin America and the ROW with respect to phenotype may be due to pediatricians from Latin America who are not familiar with MPS I and lack ready access to diagnostic methods, and therefore misclassify some patients with severe disease (Hurler) as having moderate (Hurler-Scheie) disease. In this regard, Latin America also had a higher proportion of patients with an undetermined phenotype than in the ROW (22 versus 6\%). The age of diagnosis and of symptom onset was very young for a high proportion of Latin American patients with an unknown or undetermined phenotype, suggesting that many or most are actually Hurler patients. Thus, the discrepancy in phenotype distribution may not be as marked as it appears. Cognitive impairment is one of the main features that differentiate Hurler patients from HurlerScheie and Scheie. Had some Latin American Hurler patients been misdiagnosed as Hurler-Scheie, one might have expected to see a higher proportion of Latin American Hurler-Scheie patients with cognitive impairment. However, the frequency of cognitive impairment in Hurler-Scheie patients was similar between Latin America and ROW (33 versus $35 \%$ ). In addition, the group of patients with an unknown or undetermined diagnosis in Latin America actually had a lower frequency of cognitive impairment than in the ROW (31 versus 53\%). These findings are consistent with there being a true phenotypic difference in MPS I patients from Latin America.

It is also important to bear in mind that the population of Latin America includes ancestors of European immigrants as well as an indigenous population, which could result in a different mix of genotypes and associated phenotypes as compared to the ROW. Although in some cases genotyping may be helpful in identifying patients with Hurler syndrome (Terlato and Cox 2003), genotyping is not routinely done in Latin America, and therefore the genotype data currently available in the MPS I Registry are too sparse to draw any conclusions. In the future, genotyping of Latin American patients may be helpful in further elucidating the 
Fig. 4 Prevalence of MPS I-related symptoms by region and phenotype
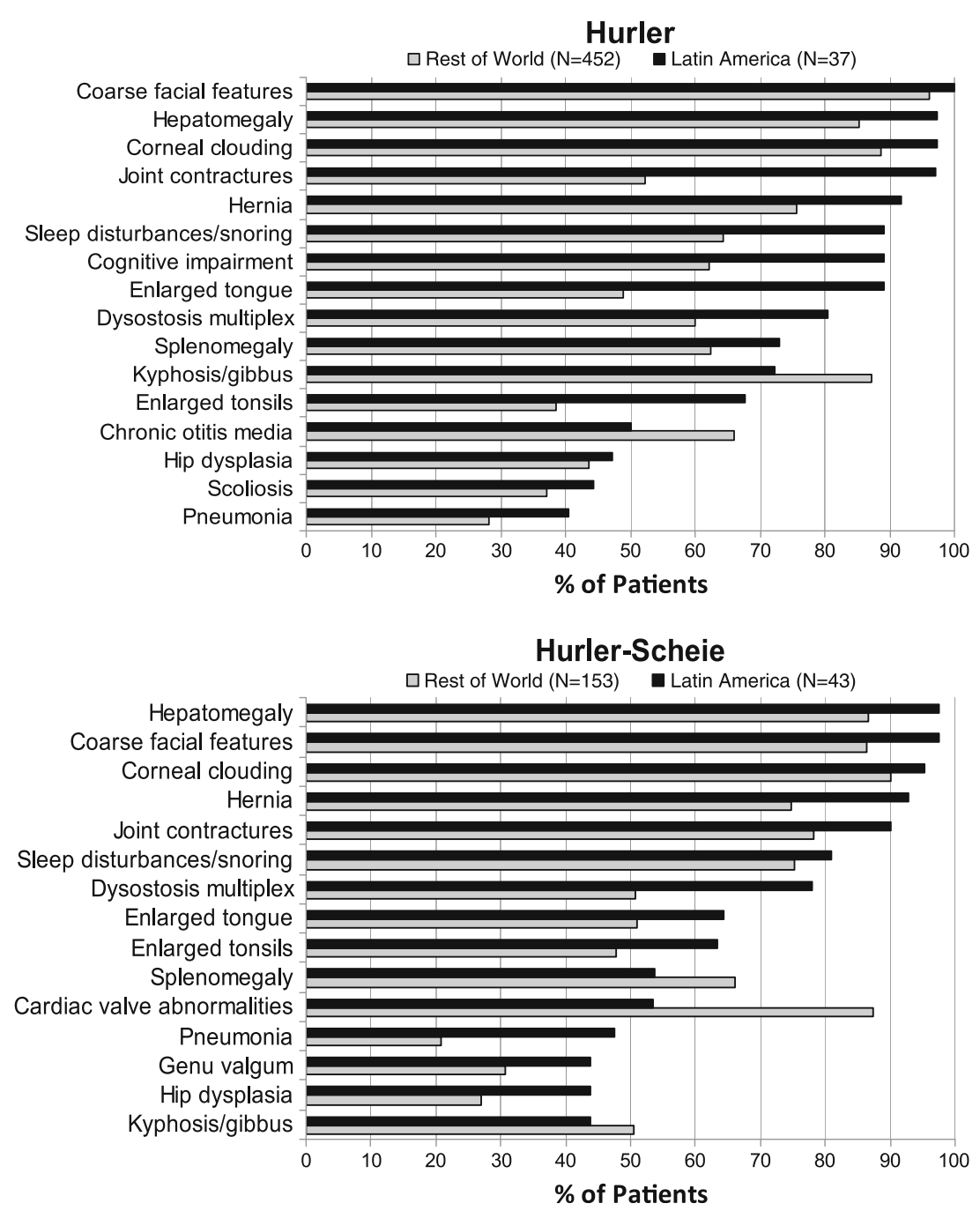

phenotype distribution as certain genotype-phenotype correlations may allow the prediction of a patient's clinical phenotype from the genotype. As genotyping becomes more common in the future, this topic will be an interesting avenue to pursue.

The different age at symptom onset for Hurler-Scheie patients may indicate regional variations in conventions for assigning phenotype to MPS I. Apparent differences in the prevalence of certain symptoms may indicate regional phenotype differences but may also be closely related to regional practices in evaluating, diagnosing, and following MPS I patients. For instance, the higher observed rate of cognitive impairment in Latin American Hurler patients may correspond to the fact that HSCT is not performed in Hurler patients in Latin America but is a relatively common treatment option in many other countries globally. Following the same rationale, the differences reported in valvular compromise may reflect less clinical monitoring and follow-up in Latin America than in the ROW, where there are more facilities to provide more regular evaluations with echocardiograms. In addition, in some countries in Central and South America, pediatricians receive less clinical training in the diagnosis of inborn errors of metabolism, and there are a limited number of specialized centers for the diagnosis, assessment, and treatment of these diseases.

Treatment information available from the MPS I Registry shows that while the majority of Hurler patients worldwide receive HSCT, this treatment is not routinely performed in most regions in Latin America. Only one Latin American patient (phenotype unknown) in the Registry has received HSCT. The later age at diagnosis of Hurler patients in Latin America than in the ROW may be one reason why HSCT is uncommon in countries where it is available. By the time many Hurler patients are diagnosed, they are beyond the age of 2 years, and thus have missed the optimum window of time for a successful transplant. In these cases ERT is likely administered to provide somatic benefits and to manage the other non-CNSrelated complications of the disease. 
Fig. 4 (continued)
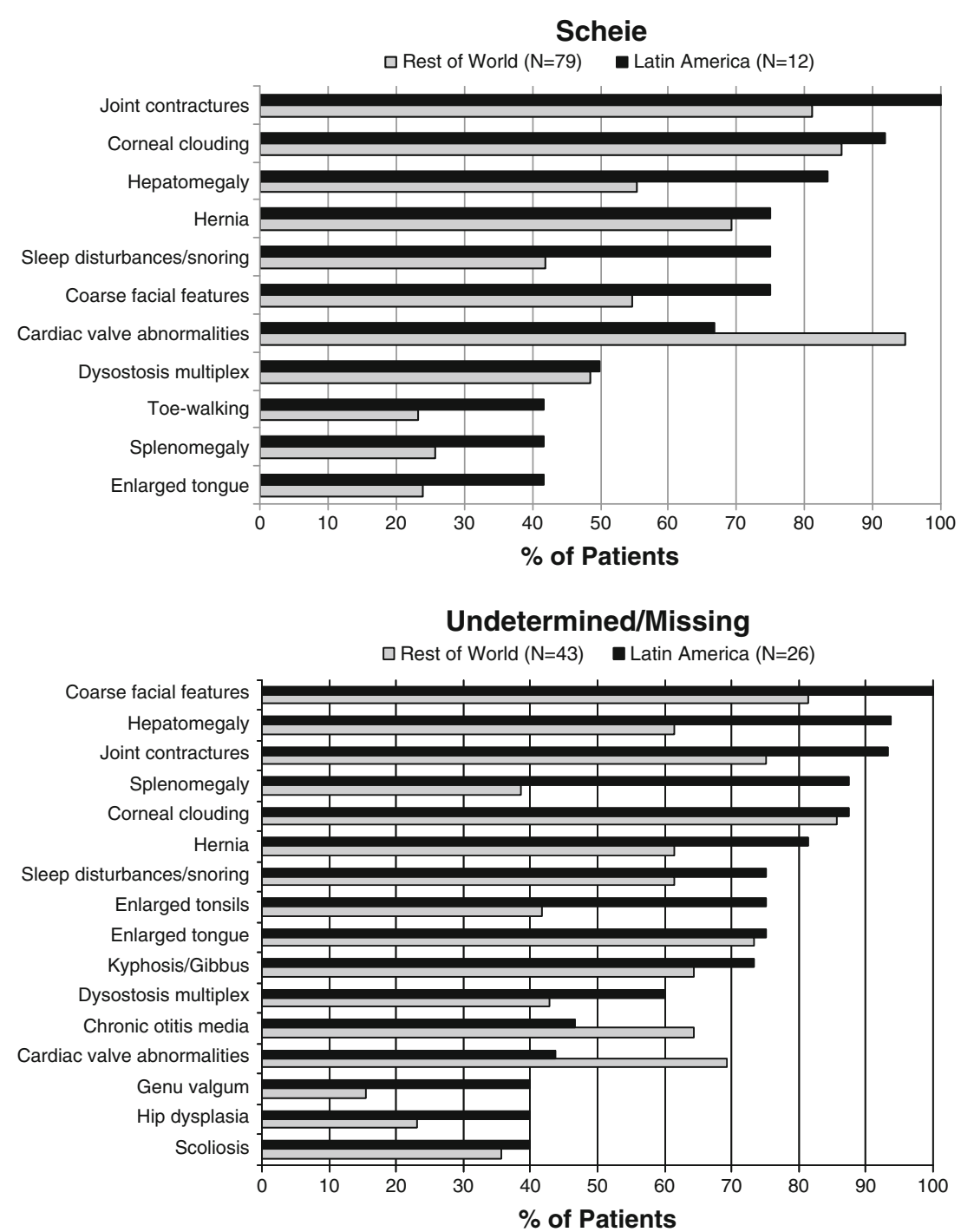

Overall, 19\% of Latin American patients and 13\% of the ROW patients received no disease-specific treatment. Some of these patients may have been diagnosed before laronidase became commercially available, and some patients may live in regions with limited access to treatment.
Furthermore, patients with delayed diagnosis and severe neurological involvement may elect to receive palliative care instead of some of the specific treatments. Further analyses of treatment choice and time to treatment using data from the MPS I Registry should account for the time
Fig. 5 Treatment status by MPS I phenotype and region

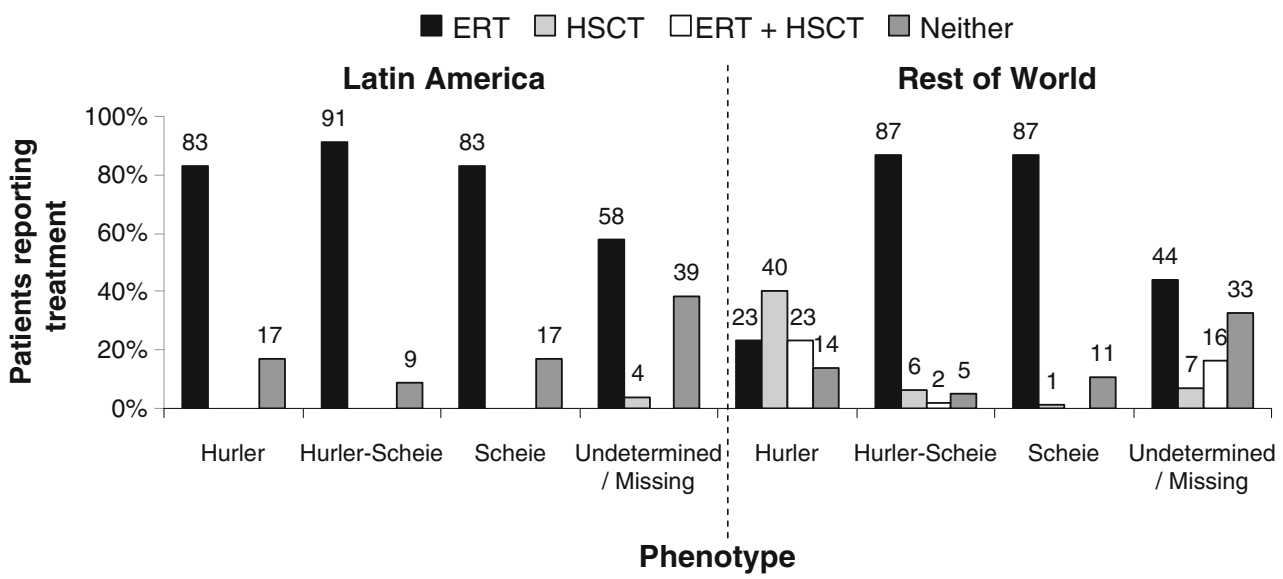


period in which patients are diagnosed and the availability of ERT and HSCT as treatment options. Further information is required to better establish optimum treatment management for MPS I patients irrespective of locality but taking into account real local treatment options. In this sense, management guidelines developed in Latin American countries (Martins et al. 2009) are of major importance, and such guidelines should be reconsidered periodically to reflect local conditions.

The fact that MPS I is a progressive disease with variable rates of irreversible features, disease awareness, or lack thereof has important implications for the effectiveness of treatment. As mentioned above, the best outcomes for HSCT occur in patients under age 2 with normal developmental quotients at the time of transplant. Findings from a recent study in MPS I dogs treated from birth with intravenous ERT demonstrated that very early treatment could prevent the development of certain disease-related complications, e.g., skeletal and valve dysplasia, that are otherwise refractory to treatment once established (Dierenfeld et al. 2010). Gabrielli et al. reported the case of a boy with attenuated MPS I who started ERT with laronidase at 5 months of age and compared his clinical course to that of his older sibling, who did not began treatment until 5 years of age. After 5 years of treatment, the younger sibling had not developed any clinical manifestations of MPS I except for mild corneal clouding. In contrast, although many of the older sibling's clinical features had improved, some, although stabilized, had persisted (Gabrielli et al. 2010).

These findings underscore the importance of heightened disease awareness as a key component to proactive diagnosis and early treatment. Physicians must be educated on which early clinical symptoms to look for in order to be able to identify and diagnose MPS I patients in a proactive manner. Some early disease manifestations, e.g., otitis media and hernia, are very common in MPS I patients but also relatively common in the general population and would not necessarily raise clinical suspicion of MPS I. Unusual clinical signs and symptoms of MPS I that are more specific and commonly present by age 2 include coarse facial features, corneal clouding, enlarged tongue and tonsils, sleep disturbance, hepatosplenomegaly, cardiac valve abnormalities, joint contractures, dysostosis multiplex, kyphosis/gibbus, and cognitive impairment (Pastores et al. 2007). As disease awareness increases, it will be interesting to see if the observed differences in phenotype persist.

An important consideration in interpreting the data presented in this study is the voluntary and observational nature of the MPS I Registry. As with all observational studies, there exists the potential for ascertainment and reporting biases. Nonetheless, the differences in phenotype distribution, prevalence of clinical manifestations, and management practices observed between the Latin American region and the ROW raise intriguing questions that should be investigated in greater depth. The findings in this study, together with further data accumulated through the MPS I Registry on an ongoing basis, provide support towards the development of a better understanding of disease progression and management in Latin America.

\section{Conclusion}

The results of this study suggest a different phenotype distribution of MPS I patients from Latin America than in the ROW. Apparent differences in the age of symptom onset and prevalence of certain symptoms may indicate regional phenotype differences but may also be related to regional practices in evaluating, diagnosing, and following MPS I patients. These data should be confirmed with larger patient numbers, which will provide additional information to support a better understanding of MPS I disease progression and management in Latin America versus the ROW.

Acknowlegments The authors thank the patients and physicians who participate in the MPS I Registry from the Latin America region as well as worldwide. The authors also acknowledge the contributions of the MPS I Registry team at Genzyme Corporation, which provided support for medical writing (Laura Artiles-Carloni) and data analyses (Jessica Kong, Lakshmi Rangachari, Gregory Fagan, and Asif Karbhari).

Competing interests The authors declare independence from the sponsors and declare that the content of the article has not been influenced by the sponsors.

Open Access This article is distributed under the terms of the Creative Commons Attribution Noncommercial License which permits any noncommercial use, distribution, and reproduction in any medium, provided the original author(s) and source are credited.

\section{References}

Aldenhoven M, Boelens JJ, de Koning TJ (2008) The clinical outcome of Hurler syndrome after stem cell transplantation. Biol Blood Marrow Transplant 14:485-498

Boelens JJ, Wynn RF, O'Meara A et al (2007) Outcomes of hematopoietic stem cell transplantation for Hurler's syndrome in Europe: a risk factor analysis for graft failure. Bone Marrow Transplant 40:225-233

Clarke L, Wraith JE, Beck M et al (2009) Long-term efficacy and safety of laronidase in the treatment of mucopolysaccharidosis I. Pediatrics 123:229-240

Cox-Brinkman J, Boelens JJ, Wraith JE et al (2006) Haematopoietic cell transplantation (HCT) in combination with enzyme replacement therapy (ERT) in patients with Hurler syndrome. Bone Marrow Transplant 38:17-21 
Dierenfeld AD, McEntee MF, Vogler CA, et al. (2010) Replacing the enzyme a-L-iduronidase at birth ameliorates symptoms in the brain and periphery of dogs with mucopolysaccharidosis type I. Sci Transl Med 2: 60ra89

Gabrielli O, Clarke LA, Bruni S, Coppa GV (2010) Enzyme-replacement therapy in a 5-month-old boy with attenuated presymptomatic MPS I: 5-year follow-up. Pediatrics 125:e183-e187

Kakkis ED, Muenzer J, Tiller GE et al (2001) Enzyme-replacement therapy in mucopolysaccharidosis I. N Engl J Med 344:182-188

Khanna G, Van Heest AE, Agel J et al (2007) Analysis of factors affecting development of carpal tunnel syndrome in patients with Hurler syndrome after hematopoietic cell transplantation. Bone Marrow Transplant 39:331-334

Martins AM, Dualibi AP, Norato D et al (2009) Guidelines for the management of mucopolysaccharidosis type I. J Pediatr 155:S32-S46

Muenzer J, Wraith JE, Clarke LA (2009) Mucopolysaccharidosis I: management and treatment guidelines. Pediatrics 123:19-29

Neufeld EF, Muenzer J (2001) The mucopolysaccharidoses. In: Scriver C, Beaudet A, Sly W, Valle D, Childs B, Kinzler K, Vogelstein B (eds) The metabolic and molecular bases of inherited disease. McGraw Hill, New York, pp 3421-3452

Pastores G, Arn P, Beck M et al (2007) The MPS I registry: design, methodology, and early findings of a global disease registry for monitoring patients with mucopolysaccharidosis type I. Mol Genet Metab 91:37-47

Sifuentes M, Doroshow R, Hoft R et al (2007) A follow-up study of MPS I patients treated with laronidase enzyme replacement therapy for 6 years. Mol Genet Metab 90:171-180
Staba SL, Escolar ML, Poe M et al (2004) Cord-blood transplants from unrelated donors in patients with Hurler's syndrome. N Engl J Med 350:1960-1969

Terlato NJ, Cox GF (2003) Can mucopolysaccharidosis type I disease severity be predicted based on a patient's genotype? A comprehensive review of the literature. Genet Med 5:286-294

Thomas JA, Jacobs S, Kierstein J, Van Hove J (2006) Outcome after three years of laronidase enzyme replacement therapy in a patient with Hurler syndrome. J Inherit Metab Dis 29:762

Thomas JA, Beck M, Clarke JTR, Cox GF (2010) Childhood onset of Scheie syndrome, the attenuated form of mucopolysaccharidosis I. J Inherit Metab Dis 33:421-427

Valstar MJ, Ruijter GJ, van Diggelen OP, Poorthuis BJ, Wijburg FA (2008) Sanfilippo syndrome: a mini-review. J Inherit Metab Dis 3:240-252

Vijay S, Wraith JE (2005) Clinical presentation and follow-up of patients with the attenuated phenotype of mucopolysaccharidosis type I. Acta Paediatr 94:872-877

Wraith JE (2005) The first 5 years of clinical experience with laronidase enzyme replacement therapy for mucopolysaccharidosis I. Expert Opin Pharmacother 6:489-506

Wraith JE, Clarke LA, Beck M et al (2004) Enzyme replacement therapy for mucopolysaccharidosis I: a randomized, doubleblinded, placebo-controlled, multinational study of recombinant human alpha-L-iduronidase (laronidase). J Pediatr 144:581-588

Wraith EJ, Hopwood JJ, Fuller M, Meikle PJ, Brooks DA (2005) Laronidase treatment of mucopolysaccharidosis I. BioDrugs $19: 1-7$ 